\title{
Defining Prolonged Length of Acute Care Stay for Surgically and Conservatively Treated Patients with Spontaneous Intracerebral Hemorrhage: A Population-Based Analysis
}

\author{
Marco Stein, ${ }^{1}$ Björn Misselwitz, ${ }^{2}$ Gerhard F. Hamann, ${ }^{3}$ Malgorzata A. Kolodziej, \\ Marcus H. T. Reinges, ${ }^{1}$ and Eberhard Uhl ${ }^{1}$ \\ ${ }^{1}$ Department of Neurosurgery, Justus-Liebig University Giessen, Klinikstrasse 33, 35392 Giessen, Germany \\ ${ }^{2}$ Institute of Quality Assurance Hesse, Frankfurter Strasse 10-14, 65760 Eschborn, Germany \\ ${ }^{3}$ Department of Neurology and Neurological Rehabilitation, Bezirkskrankenhaus Günzburg, Ludwig-Heilmeyer-Strasse 2, \\ 89312 Günzburg, Germany
}

Correspondence should be addressed to Marco Stein; dr.stein@email.de

Received 1 December 2015; Revised 9 February 2016; Accepted 7 March 2016

Academic Editor: Andrew Ducruet

Copyright (C) 2016 Marco Stein et al. This is an open access article distributed under the Creative Commons Attribution License, which permits unrestricted use, distribution, and reproduction in any medium, provided the original work is properly cited.

\begin{abstract}
Background. The definition of prolonged length of stay (LOS) during acute care remains unclear among surgically and conservatively treated patients with intracerebral hemorrhage (ICH). Methods. Using a population-based quality assessment registry, we calculated change points in LOS for surgically and conservatively treated patients with ICH. The influence of comorbidities, baseline characteristics at admission, and in-hospital complications on prolonged LOS was evaluated in a multivariate model. Results. Overall, 13272 patients with ICH were included in the analysis. Surgical therapy of the hematoma was documented in 1405 (10.6\%) patients. Change points for LOS were 22 days (CI: 8, 22; CL 98\%) for surgically treated patients and 16 days (CI: 16, 16; CL: 99\%) for conservatively treated patients. Ventilation therapy was related to prolonged LOS in surgically (OR: 2.2, 95\% CI: 1.5-3.1; $P<0.001$ ) and conservatively treated patients (OR: $2.5,95 \% \mathrm{CI}: 2.2-2.9 ; P<0.001$ ). Two or more in-hospital complications in surgical patients (OR: 2.7, 95\% CI: 2.1-3.5) and $\geq 1$ in conservative patients (OR: 3.0, 95\% CI: 2.7-3.3) were predictors of prolonged LOS. Conclusion. The definition of prolonged LOS after ICH could be useful for several aspects of quality management and research. Preventing in-hospital complications could decrease the number of patients with prolonged LOS.
\end{abstract}

\section{Introduction}

Between 10 and $20 \%$ of all strokes are caused by spontaneous intracerebral hemorrhage (ICH) [1]. Spontaneous ICH is associated with significant morbidity and mortality worldwide. Length of hospital stay (LOS) is a factor directly related to hospital costs and is influenced by different variables [2]. In-hospital complications after stroke were known to be associated with longer LOS [3]. However, most available stroke data about in-hospital complications concerns ischemic stroke and few publications focus on ICH $[4,5]$. Data about prolonged LOS after ICH and influencing factors were only available for a small Asian cohort [6]. Currently, no population-based data about the definition of prolonged LOS with a focus on ICH exists in Europe. Furthermore, in the available publications on this topic, no difference was made between surgically and conservatively treated patients with ICH [7]. In this context, the differentiation between ICH patients with and without ventilation therapy is important and insufficiently depicted in the available publications. However, data about this topic are needed for the improvement of quality assessment, for future research, and, last but not least, for the development of adequate and realistic remuneration systems for patients with ICH.

Data for LOS are shown in timelines highlighting potential change points characterized by changes in data distribution or data spread. These change points are often essential for data interpretation. Change-point analysis also has the power to detect and determine these change points retrospectively, as well as to test them for statistical significance. 
Today, change-point analyses are widely used in engineering, economics, and natural sciences [8-10].

The purpose of this study was to evaluate LOS among surgically and conservatively treated patients with spontaneous ICH during acute care. We set out to investigate various aspects associated with LOS that include comorbidities, inhospital complications, ventilation therapy, and patients' baseline characteristics. Using change-point analysis, we aimed to calculate different change points for a prolonged LOS for surgically and conservatively treated patients.

\section{Methods}

The dataset for this study was obtained from a large prospective stroke database in the state of Hesse, Germany. The Hessian Stroke Registry is mandatory for all stroke patients diagnosed with ischemic stroke (ICD-10: I63), ICH (ICD-10: I61), and subarachnoid hemorrhage (ICD-10: I60). For this study we only included patients diagnosed with spontaneous ICH (ICD-10: I61.0 to I61.9), with an admission date between January 1, 2007, and December 31, 2014.

The term "pre-ICH disability" was defined as a modified Rankin Scale (mRS) of 3-5 prior to ICH onset. Initial Glasgow Coma Score (GCS) was assessed at hospital admission. In-hospital complications were recorded during the acute care stay and were categorized in 1,2 , and $\geq 3$ secondary complications.

The basis for the data registration in the Hessian stroke database was regulated by the German Social Code, Book Five. Due to the anonymous data collection for this study, neither institutional board approval nor informed consent was required.

2.1. Statistical Methods. For the comparison of categorical variables, the chi-square test was used. Continuous variables were analysed by the Student $t$-test or the MannWhitney $U$ Test. We analysed the relation between preICH existing comorbidities, baseline characteristics, and inhospital complications to a prolonged LOS for surgically and conservatively treated patients in a multivariate regression model. Variables with a $P$ value $<0.1$ in a univariate analysis were included in the multivariate analysis. Odds ratios (OR) and $95 \%$ confidence intervals (CI) were calculated to observe the relation between the quantity of in-hospital complications and the chance of a prolonged LOS for surgically and conservatively treated patients. These analyses were performed with SPSS 21 for Windows (IBM Inc., Armonk, NY, USA). Cut-off values for the duration of ventilation therapy were calculated by receiver operating characteristic (ROC) analysis with MedCal Vers.15.8 for Windows (MedCalc Software, Ostend, Belgium). Youden's index (sensibility+ specificity-1) and the area under the curve (AUC) were calculated. The highest available Youden's index was defined as the best cutoff point for the duration of prolonged LOS in surgically and conservatively treated patients.

2.2. Change-Point Analysis. The cumulative sums (CUSUM) were calculated by the method of Taylor [11]. Sudden turns in the CUSUM curve direction represent changes in data average. A CI for every change point was calculated. The closer a $\mathrm{CI}$ is, the more accurate the time of the change point can be pinpointed. The confidence level (CL) shows how frequently the calculated change point will likely occur. Further information provided by change-point analysis is the level of the calculated change point. A level of 1 indicates a high importance of the change. In our analysis, only levels 1 and 2 changes were accepted to be included. If outliers in the LOS data timeline led to a violation of independent error assumption, the group rows of the change-point analysis were compared to groups of two rows.

\section{Results}

3.1. Basic Characteristics. Overall, 13292 patients with the diagnosis of spontaneous ICH were identified in the study period. In 20 patients $(0.2 \%)$, LOS data were missing. The remaining 13272 patients were included in the analysis. Median age of the patients was 67 years (range: $19-93$ years). Approximately half of the cohort, 6376 patients (48.0\%), was female. Median GCS was 12 (IQR: 7-15). Secondary expansion of the ICH into the ventricles was observed in 1777 patients (13.4\%). Preexisting comorbidities like arterial hypertension, diabetes, hypercholesterolemia, atrial fibrillation, prior insult, and other comorbidities were observed in 9799 (73.8\%), 2045 (15.4\%), 1687 (12.7\%), 2426 (18.3\%), 2039 (15.4\%), and 3199 (24.1\%) of the patients, respectively. A pre-ICH disability was documented in 2062 patients (15.4\%). During the acute care stay, operations of the hematoma were performed in $1405(10.6 \%)$ patients. Ventilation therapy in the intensive care unit was documented in 3438 (25.9\%) patients. The median ventilation time was four days (IQR: 1-13). Inhospital complications that occurred with an incidence $>1 \%$ are listed in Table 1 . Overall, $37.1 \%$ of patients $(N=4927)$ had one or more complications during acute care stay. The total in-hospital mortality rate was $23.6 \%$ (3138 patients), and the median mRS of the survivors at hospital discharge was four with an IQR of two to five.

3.2. The Calculation of Prolonged Intensive Care Stay. The calculated change points for a prolonged LOS at acute care were 22 days (CI: 8, 22; CL 98\%) for surgically treated patients and 16 days (CI: 16, 16; CL: 99\%) for conservatively treated patients. The CUSUM charts by days of acute care stay are shown in Figures 1(a) and 1(b). A subgroup analysis by ventilation therapy for surgically and conservatively treated patients is listed in Table 2 .

3.3. Comparing Surgical and Conservative Patients. The rate of patients with ventilation therapy in the ICU was increased in surgically treated patients compared to conservatively treated patients (1060 patients $(75.4 \%)$ versus 2378 patients (20.0); $P<0.001)$. Median acute care stay after ICH was significantly longer in surgically treated patients compared to conservatively treated patients (15 days, IQR: 8-23, versus 9 days, IQR: $3-14 ; P<0.001)$. By defining prolonged LOS as $>22$ days for surgically treated patients and $>16$ days for conservatively treated patients, $366(26.0 \%)$ patients of the surgical group and 2289 (19.3\%) patients of the conservative 


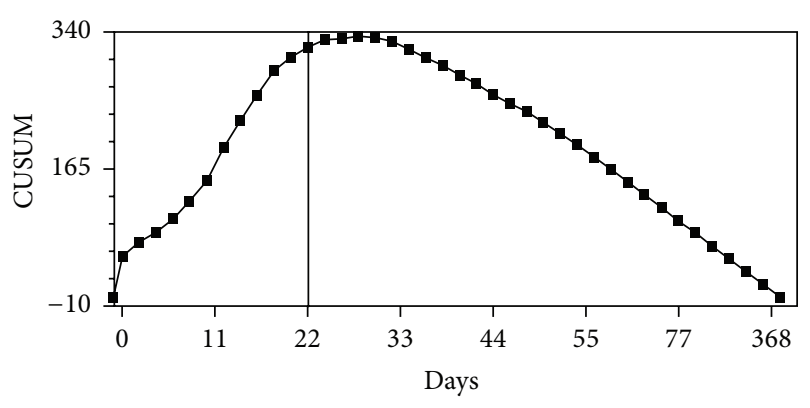

(a)

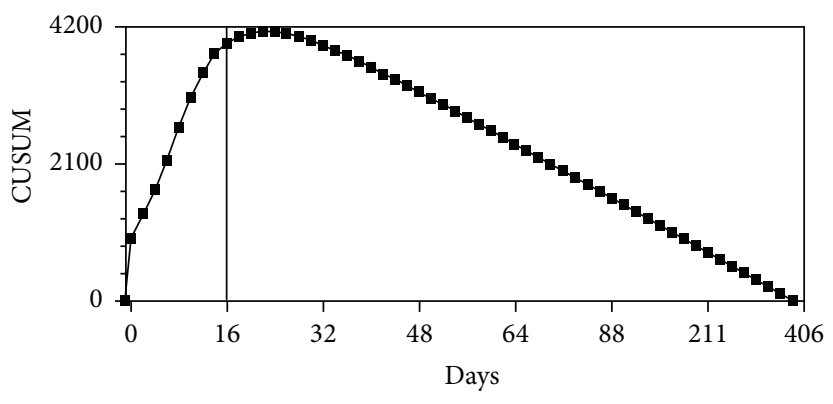

(b)

Figure 1: Timelines of cumulative sum control (CUSUM) analysis. CUSUM analysis by days of acute care stay for surgically (a) and conservatively treated (b) patients. The calculated change points were 22 days for surgically treated patients and 16 days for conservatively treated patients with spontaneous intracerebral hemorrhage.

TABLE 1: In-hospital complications for patients with spontaneous intracerebral hemorrhage.

\begin{tabular}{lc}
\hline$N(\%)$ & $13272(100.0)$ \\
Pulmonary & $1981(14.9)$ \\
Brain edema & $823(6.2)$ \\
Urogenital & $767(5.8)$ \\
Cardial & $756(5.7)$ \\
Hydrocephalus & $554(4.2)$ \\
Epilepsy & $488(3.7)$ \\
Rebleeding & $453(3.4)$ \\
Sepsis & $231(1.7)$ \\
Brain infarction & $178(1.3)$ \\
Other & $1661(12.5)$ \\
\hline
\end{tabular}

(Only secondary complications with an occurrence $>1 \%$ are listed.)

group with prolonged LOS were identified. Differences in prior ICH comorbidities, baseline characteristics, and inhospital complications for prolonged and nonprolonged LOS for both groups are shown in Table 3 .

Ventilation therapy was associated with prolonged LOS in a univariate analysis for surgically and conservatively treated patients. Not only did the quantity of patients with ventilation therapy increase, but the duration of ventilation therapy was also longer in patients with prolonged LOS when compared to nonprolonged LOS.

In an ROC analysis of ventilation therapy for surgically treated patients, we observed an LOS of $>9$ days (Youden's index: 0.646; AUC: 0.889, 95\% CI: 0.875-0.901; $P<0.001)$ as the optimal cut-off point to predict prolonged LOS. For conservatively treated patients, the optimal cut-off point of ventilation therapy was detected at $>17$ days (Youden's index: 0.489; AUC: 0.821, 95\% CI: 0.796-0.843; $P<0.001)$.

3.4. Binary Regression Analysis. The main predictors of prolonged LOS for surgically and conservatively treated patients are shown in Table 4 . In the surgical group, the presence of two or more in-hospital complications and ventilation therapy were identified as independent predictors of prolonged LOS. The observed age and GCS were lower in surgically treated patients with prolonged LOS compared to patients without prolonged LOS. GCS was no longer a significant predictor after adjustment for age, ventilation therapy, and $\geq 2$ in-hospital complications. In the conservative group, at least one in-hospital complication and ventilation therapy were independently associated with prolonged LOS. Patients with a prolonged LOS were younger compared to patients without prolonged LOS. The observed GCS was slightly higher in patients with a prolonged LOS (Table 3 ) compared to patients with nonprolonged LOS.

\section{Discussion}

4.1. Summary of Findings. With the definition of prolonged LOS as $>22$ days for surgically treated patients and $>16$ days for conservatively treated patients after spontaneous ICH, every fourth patient in the surgical, and approximately every fifth patient in the conservative group, shows a prolonged LOS during acute care in Central Germany. No representative statement of prolonged or nonprolonged LOS can be made when only observing the median or mean LOS. To the best of our knowledge, this is the first definition of prolonged LOS for surgically and conservatively treated patients after spontaneous ICH. Furthermore, change points for prolonged LOS were higher among surgically treated and ventilated patients (22 days; CI: 18-22), compared to conservatively treated patients with ventilation therapy (10 days; CI: 6-10). Moreover, the calculated change points for LOS of nonventilated patients were higher in surgically treated patients (19 days; CI: 15-21) when compared to conservatively treated patients (>16 days; CI 10-16). In this context, the majority of patients in the surgical group were ventilated and the majority of conservatively treated patients were nonventilated. This explains that the same change points for these groups with different CI and CL were detected.

Publications on the topic of prolonged LOS are rare, especially for patients with ICH [6]. Most publications that evaluate prolonged LOS after stroke exclusively depict ischemic stroke [12] or define prolonged acute care stay with a scoring system without differentiation for ischemic stroke or intracerebral hemorrhage or for patients with and without ventilation therapy [13]. The association between 
TABLE 2: Results of a change-point analysis for prolonged length of stay (LOS). Median LOS and calculated change points in LOS timeline by surgical and conservative treatment, both with and without ventilation therapy.

\begin{tabular}{|c|c|c|c|c|c|}
\hline Patients & $\begin{array}{c}\text { Days } \\
\text { (Median) }\end{array}$ & $\begin{array}{l}\text { Days } \\
(\mathrm{IQR})^{*}\end{array}$ & $\begin{array}{c}\text { Prolonged LOS } \\
\text { (Change point, days) }\end{array}$ & Confidence interval & $\begin{array}{c}\text { Confidence } \\
\text { level }\end{array}$ \\
\hline $\begin{array}{l}\text { Conservative } \\
(N=11867)\end{array}$ & 9 & $3-14$ & 16 & 16,16 & $99 \%$ \\
\hline $\begin{array}{l}\text { Surgical } \\
(N=1405)\end{array}$ & 15 & $8-23$ & 22 & 8,22 & $98 \%$ \\
\hline $\begin{array}{l}\text { Ventilation } \\
\text { Conservative } \\
(N=2378)\end{array}$ & 6 & $1-20$ & 10 & 6,10 & $98 \%$ \\
\hline $\begin{array}{l}\text { Ventilation } \\
\text { Surgical } \\
(N=1060) \\
\end{array}$ & 16 & $9-25$ & 22 & 18,22 & $99 \%$ \\
\hline $\begin{array}{l}\text { Nonventilated } \\
\text { Conservative } \\
(N=9489) \\
\end{array}$ & 9 & $4-14$ & 16 & 10,16 & $95 \%$ \\
\hline $\begin{array}{l}\text { Nonventilated } \\
\text { Surgical }(N=345)\end{array}$ & 12 & $2-17$ & 19 & 15,21 & $100 \%$ \\
\hline
\end{tabular}

*Interquartile range (P25-P75).

TABLE 3: Main characteristics of 13272 patients with spontaneous intracerebral hemorrhage (ICH). Baseline characteristics are presented by prolonged and nonprolonged length of stay (LOS) for surgical and conservative treatment.

\begin{tabular}{|c|c|c|c|c|c|c|}
\hline \multirow[b]{2}{*}{$N(\%)$} & \multicolumn{3}{|c|}{ Surgical group } & \multicolumn{3}{|c|}{ Conservative group } \\
\hline & $\begin{array}{c}\text { Nonprolonged } \\
\text { LOS ( } \leq 22 \text { days }) \\
N=1039\end{array}$ & $\begin{array}{c}\text { Prolonged } \\
\text { LOS (>22 days) } \\
N=366 \\
\end{array}$ & $P$ value & $\begin{array}{c}\text { Nonprolonged } \\
\text { LOS ( } \leq 16 \text { days }) \\
N=9578 \\
\end{array}$ & $\begin{array}{c}\text { Prolonged } \\
\text { LOS (>16 days) } \\
N=2289\end{array}$ & $P$ value \\
\hline Female & $469(45.1)$ & $154(42.1)$ & 0.310 & $4726(49.3)$ & $1027(44.9)$ & $<0.001$ \\
\hline Age $^{*}$ & $68(56-75)$ & $66(52-74)$ & 0.006 & $76(67-83)$ & $73(63-80)$ & $<0.001$ \\
\hline Pre-ICH disability & $81(7.8)$ & $34(9.3)$ & 0.199 & $1594(16.6)$ & $353(15.4)$ & 0.170 \\
\hline GCS at admission* & $10(3-14)$ & $7(3-13)$ & 0.001 & $13(7-15)$ & $13(8-15)$ & 0.043 \\
\hline Secondary IVH & $113(10.9)$ & $40(10.9)$ & 0.978 & $1288(13.4)$ & $336(14.7)$ & 0.124 \\
\hline Ventilation therapy & $739(71.1)$ & $321(87.5)$ & $<0.001$ & $1640(17.1)$ & $738(32.2)$ & $<0.001$ \\
\hline Duration ventilation therapy, days* & $5(2-11)$ & $20(9-28)$ & $<0.001$ & $2(1-4)$ & $17(8-24)$ & $<0.001$ \\
\hline \multicolumn{7}{|l|}{ Comorbidities: } \\
\hline Arterial hypertension & $651(62.7)$ & $232(63.4)$ & 0.803 & $7134(74.5)$ & $1782(77.9)$ & 0.001 \\
\hline Diabetes mellitus & $114(11.0)$ & 43 (11.7) & 0.685 & $1507(15.7)$ & $381(16.6)$ & 0.284 \\
\hline Hypercholesterolemia & $92(8.9)$ & $29(7.9)$ & 0.585 & $1244(13.0)$ & $322(14.1)$ & 0.171 \\
\hline Atrial fibrillation & $182(17.5)$ & $65(17.8)$ & 0.916 & $1771(18.5)$ & $408(17.8)$ & 0.460 \\
\hline Prior insult & $96(9.2)$ & $32(8.7)$ & 0.777 & $1559(16.3)$ & $352(15.4)$ & 0.293 \\
\hline Other & $303(29.2)$ & $118(32.2)$ & 0.269 & $2202(23.0)$ & $576(25.2)$ & 0.027 \\
\hline \multicolumn{7}{|l|}{ In-hospital complications: } \\
\hline 1 complication & $318(30.6)$ & $131(35.8)$ & 0.067 & $2130(22.2)$ & $724(31.6)$ & $<0.001$ \\
\hline 2 complications & $145(14.0)$ & $101(27.6)$ & $<0.001$ & $539(5.6)$ & $379(16.6)$ & $<0.001$ \\
\hline$\geq 3$ complications & $71(6.8)$ & $64(17.5)$ & $<0.001$ & $143(1.5)$ & $182(8.0)$ & $<0.001$ \\
\hline In-hospital mortality & $167(16.1)$ & $20(5.5)$ & $<0.001$ & $2775(29.0)$ & $176(7.7)$ & $<0.001$ \\
\hline Length of hospital stay, days* & $12(5-16)$ & $31(26-43)$ & $<0.001$ & $7(2-11)$ & $23(19-29)$ & $<0.001$ \\
\hline
\end{tabular}

${ }^{*}$ Median and IQR (P25-P75). 
TABLE 4: Predictors of prolonged length of stay (LOS) during acute care. Results for the prediction of prolonged LOS in a binary logistic regression model are shown by surgical and conservative treatment.

\begin{tabular}{lccc}
\hline Predictor & OR $(95 \%$ CI $)$ & Adjusted OR $(95 \%$ CI $)$ & $P$ value \\
\hline & Surgical group $(N=1405)$ & & 0.011 \\
Age & $0.988(0.980-0.996)$ & $0.988(0.980-0.997)$ & 0.384 \\
GCS & $0.958(0.933-0.983)$ & $2.176(1.511-3.136)$ & $<0.001$ \\
Ventilation therapy & $2.896(2.062-4.067)$ & $2.701(2.077-3.512)$ & $<0.001$ \\
$\geq 2$ in-hospital complications & $3.128(2.425-4.035)$ & & \\
\hline & Conservative group $(N=11867)$ & & \\
Age & $0.987(0.983-0.990)$ & $1.078(1.063-1.092)$ & $<0.001$ \\
GCS & $1.011(1.000-1.021)$ & $2.505(2.193-2.861)$ & $<0.001$ \\
Ventilation therapy & $2.303(2.079-2.552)$ & $2.986(2.701-3.300)$ & $<0.001$ \\
$\geq 1$ in-hospital complications & $3.080(2.805-3.381)$ & $1.231(1.094-1.386)$ & $1.023(0.915-1.145)$ \\
Arterial hypertension & $1.204(1.080-1.343)$ & & 0.001 \\
Other comorbidities & $1.126(1.013-1.252)$ & 0.687 \\
\hline
\end{tabular}

comorbidities and LOS for stroke patients was controversial in several studies [13-16]. In our data, the presence of arterial hypertension was only associated with prolonged LOS for conservatively treated patients. Stroke severity is a predictor of prolonged LOS in stroke patients $[13,14]$. However, these publications are not specifically focused on patients with $\mathrm{ICH}$ and represent mixed cohorts of ischemic stroke and $\mathrm{ICH}$, and no differentiations in the calculation of prolonged LOS between surgical and conservative treatment were made.

Severity of ICH was documented with the GCS at admission in our data, and a lower GCS was associated with prolonged LOS of surgically treated patients in univariate analysis. After adjustment for several predictors in a binary logistic regression model (Table 4), GCS no longer remains a predictor of prolonged LOS. In-hospital complications are a known predictor of LOS in stroke patients [3] and in patients with ICH [17]. In a previous publication of our study group, two or more in-hospital complications in surgically treated patients and at least one or more in-hospital complications in conservatively treated patients were associated with higher rates of in-hospital mortality [5]. In the current analysis on prolonged LOS, the accumulation of two or more inhospital complications in the surgically treated group and the presence of at least one or more in-hospital complications in the conservative group were related to higher chance of prolonged LOS during acute care stay. Ventilation therapy was also a predictor of prolonged LOS [18]. In line with previous studies, ventilation therapy was an important and unsurprising predictor of prolonged LOS, independent of the treatment form in our data. Furthermore, we were able to calculate significant cut-off values for the duration of ventilation therapy by ROC analysis.

4.2. Strengths and Limitations. The strengths of our study are the large cohort of patients, the availability of prospectively collected detailed data, the method of change-point analysis, the analysis of prolonged LOS for several conditions (surgical and conservative treatment, both with and without ventilation therapy), and the population-based design. However, some limitations of our study should be discussed. Our study was not a randomized trial for surgical treatment of intracerebral hematomas, and the indication for surgery was not standardized. In the used database, hematoma volumes of the patients were not available. Hematoma volume is a known predictor of mortality and could influence the course of acute care stay. However, several publications observed a similar predictive value of the GCS at admission alone compared to scoring systems that include hematoma volume [19-21]. Furthermore, hematoma volume was not a predictor of prolonged LOS in a previous study [6].

Patients with ICH may experience several medical complications during acute care stay; in the used database not all possible complications are listed in detail (e.g., hyperglycemia). However, several known important predictors and variables during acute care stay were analysed in our data such as comorbidities, age, gender, GCS, the presence of secondary IVH, duration of ventilation therapy, in-hospital complications, in-hospital mortality, and LOS.

\section{Conclusion}

For the first time, change points for LOS during acute care were defined for surgically and conservatively treated patients with ICH. Furthermore, we could identify different change points for prolonged LOS for surgically and conservatively treated patients, both with and without ventilation therapy after spontaneous ICH. The calculated values for the definition of prolonged acute care stay could be useful for several aspects of quality management, research, and planning negotiations with health insurance groups in order to develop adequate and realistic remuneration systems for the acute care therapy of patients with spontaneous ICH.

\section{Abbreviations}

AUC: Area under the curve

CI: Confidence interval

CL: Confidence level

CUSUM: Cumulative sum

GCS: Glasgow Coma Score 
ICH: Intracerebral hemorrhage

IQR: Interquartile range

IVH: Intraventricular hemorrhage

LOS: Length of stay

OR: Odds ratio

ROC: Receiver operating curve

SD: Standard deviation.

\section{Competing Interests}

The authors declare that they have no competing interests.

\section{Acknowledgments}

The participants of the Hesse Stroke Working Group, who administered data on stroke patients in the context of external quality assurance, are listed at http://www.gqhnet.de/.

\section{References}

[1] J. M. Ferro, "Update on intracerebral haemorrhage," Journal of Neurology, vol. 253, no. 8, pp. 985-999, 2006.

[2] D. A. Gruenberg, W. Shelton, S. L. Rose, A. E. Rutter, S. Socaris, and G. McGee, "Factors influencing length of stay in the intensive care unit," American Journal of Critical Care, vol. 15, no. 5, pp. 502-509, 2006.

[3] A. Ingeman, G. Andersen, H. H. Hundborg, M. L. Svendsen, and S. P. Johnsen, "In-hospital medical complications, length of stay, and mortality among stroke unit patients," Stroke, vol. 42, no. 11, pp. 3214-3218, 2011.

[4] J. S. Balami and A. M. Buchan, "Complications of intracerebral haemorrhage," The Lancet Neurology, vol. 11, no. 1, pp. 101-118, 2012.

[5] M. Stein, G. F. Hamann, B. Misselwitz, E. Uhl, M. Kolodziej, and M. H. Reinges, "In-hospital mortality and complication rates in surgically and conservatively treated patients with spontaneous intracerebral hemorrhage in Central Europe: a populationbased study," World Neurosurgery, vol. 88, pp. 306-310, 2016.

[6] C.-L. Chan, H.-W. Ting, and H.-T. Huang, "The definition of a prolonged intensive care unit stay for spontaneous intracerebral hemorrhage patients: an application with national health insurance research database," BioMed Research International, vol. 2014, Article ID 891725, 9 pages, 2014.

[7] M. Stepanova, C. Venkatesan, L. Altaweel, A. Mishra, and Z. M. Younossi, "Recent trends in inpatient mortality and resource utilization for patients with stroke in the United States: 20052009," Journal of Stroke and Cerebrovascular Diseases, vol. 22, no. 4, pp. 491-499, 2013.

[8] M. B. Nigro, S. N. Pakzad, and S. Dorvash, "Localized structural damage detection: a change point analysis," Computer-Aided Civil and Infrastructure Engineering, vol. 29, no. 6, pp. 416-432, 2014.

[9] M. Beudel, S. Little, A. Pogosyan et al., "Tremor reduction by deep brain stimulation is associated with gamma power suppression in Parkinson's disease," Neuromodulation, vol. 18, no. 5, pp. 349-354, 2015.

[10] L. Sood and A. Owen, "A 10-year service evaluation of an assertive community treatment team: trends in hospital bed use," Journal of Mental Health, vol. 23, no. 6, pp. 323-327, 2014.
[11] W. Taylor, "Change-Point Analysis: A Powerful New Tool for Detecting Change," January 2016, http://variation.com/cpa/ index.html.

[12] N. Spratt, Y. Wang, C. Levi, K. Ng, M. Evans, and J. Fisher, "A prospective study of predictors of prolonged hospital stay and disability after stroke," Journal of Clinical Neuroscience, vol. 10, no. 6, pp. 665-669, 2003.

[13] S. Koton, N. M. Bornstein, R. Tsabari, and D. Tanne, "Derivation and validation of the prolonged length of stay score in acute stroke patients," Neurology, vol. 74, no. 19, pp. 1511-1516, 2010.

[14] M. N. Diringer, D. F. Edwards, D. T. Mattson et al., "Predictors of acute hospital costs for treatment of ischemic stroke in an academic center," Stroke, vol. 30, no. 4, pp. 724-728, 1999.

[15] R. Luengo-Fernandez, A. M. Gray, and P. M. Rothwell, "Population-based study of determinants of initial secondary care costs of acute stroke in the United Kingdom," Stroke, vol. 37, no. 10, pp. 2579-2587, 2006.

[16] P. Appelros, "Prediction of length of stay for stroke patients," Acta Neurologica Scandinavica, vol. 116, no. 1, pp. 15-19, 2007.

[17] A. M. Naidech, B. R. Bendok, P. Tamul et al., "Medical complications drive length of stay after brain hemorrhage: a cohort study," Neurocritical Care, vol. 10, no. 1, pp. 11-19, 2009.

[18] C. M. Martin, A. D. Hill, K. Burns, and L. M. Chen, "Characteristics and outcomes for critically ill patients with prolonged intensive care unit stays," Critical Care Medicine, vol. 33, no. 9, pp. 1922-1936, 2005.

[19] M. Al-Khaled and J. Eggers, "Prognosis of intracerebral hemorrhage after conservative treatment," Journal of Stroke and Cerebrovascular Diseases, vol. 23, no. 2, pp. 230-234, 2014.

[20] A. R. Parry-Jones, K. A. Abid, M. Di Napoli et al., "Accuracy and clinical usefulness of intracerebral hemorrhage grading scores: a direct comparison in a UK population," Stroke, vol. 44, no. 7, pp. 1840-1845, 2013.

[21] D. B. Zahuranec, B. N. Snchez, D. L. Brown et al., "Computed tomography findings for intracerebral hemorrhage have little incremental impact on post-stroke mortality prediction model performance," Cerebrovascular Diseases, vol. 34, no. 1, pp. 8692, 2012. 


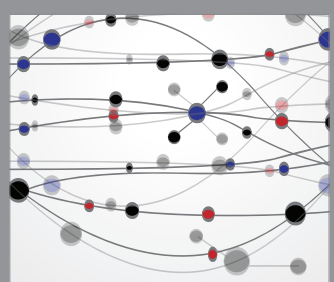

The Scientific World Journal
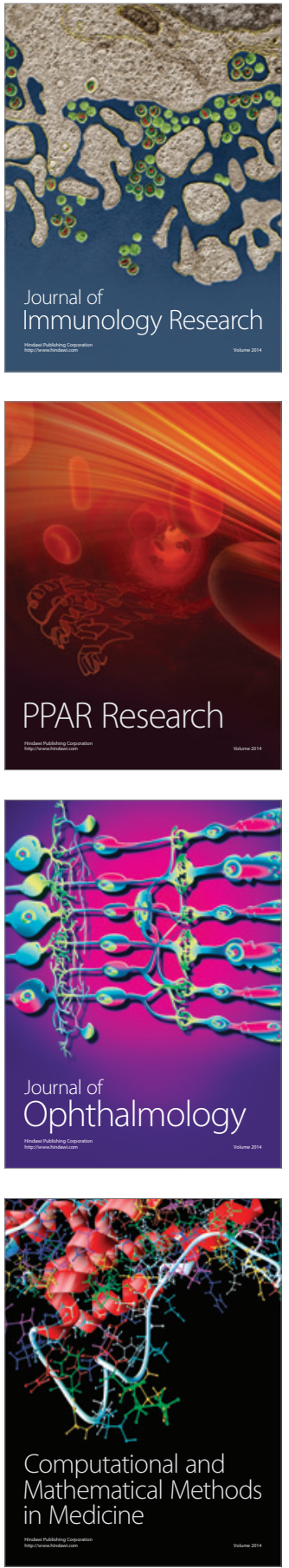

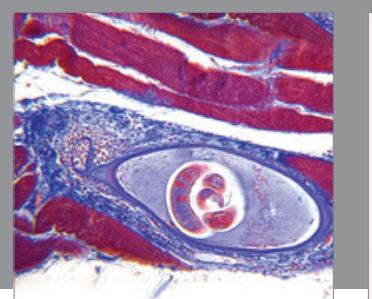

Gastroenterology Research and Practice

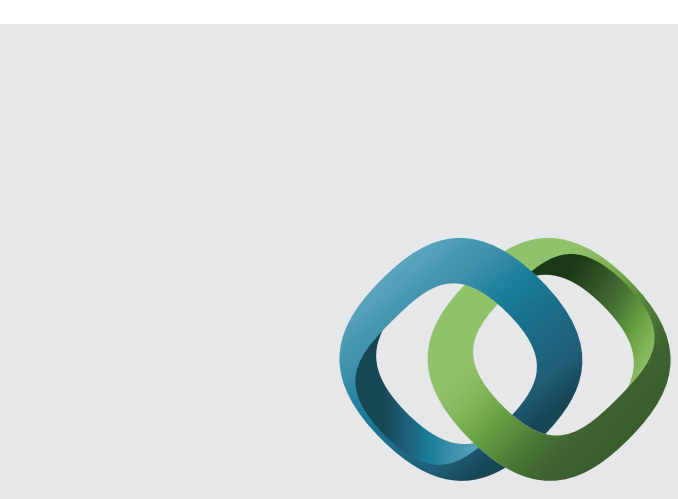

\section{Hindawi}

Submit your manuscripts at

http://www.hindawi.com
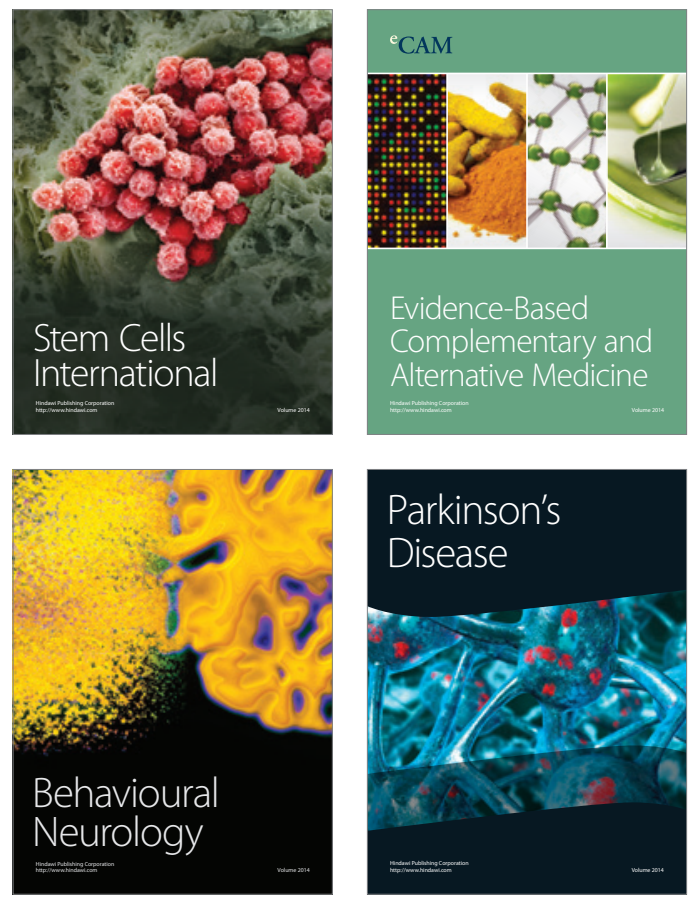
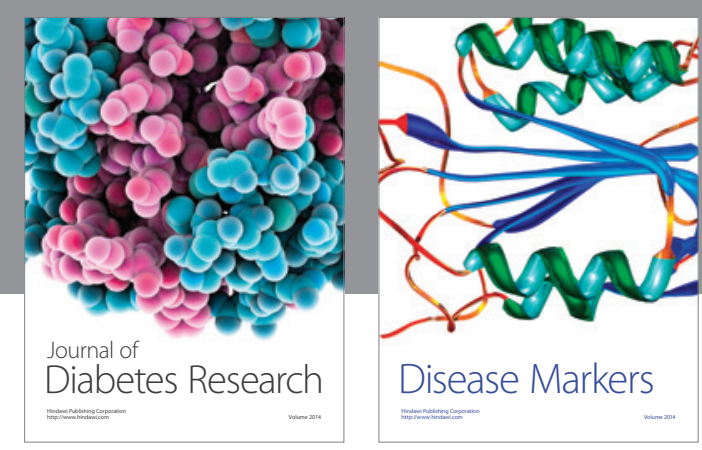

Disease Markers
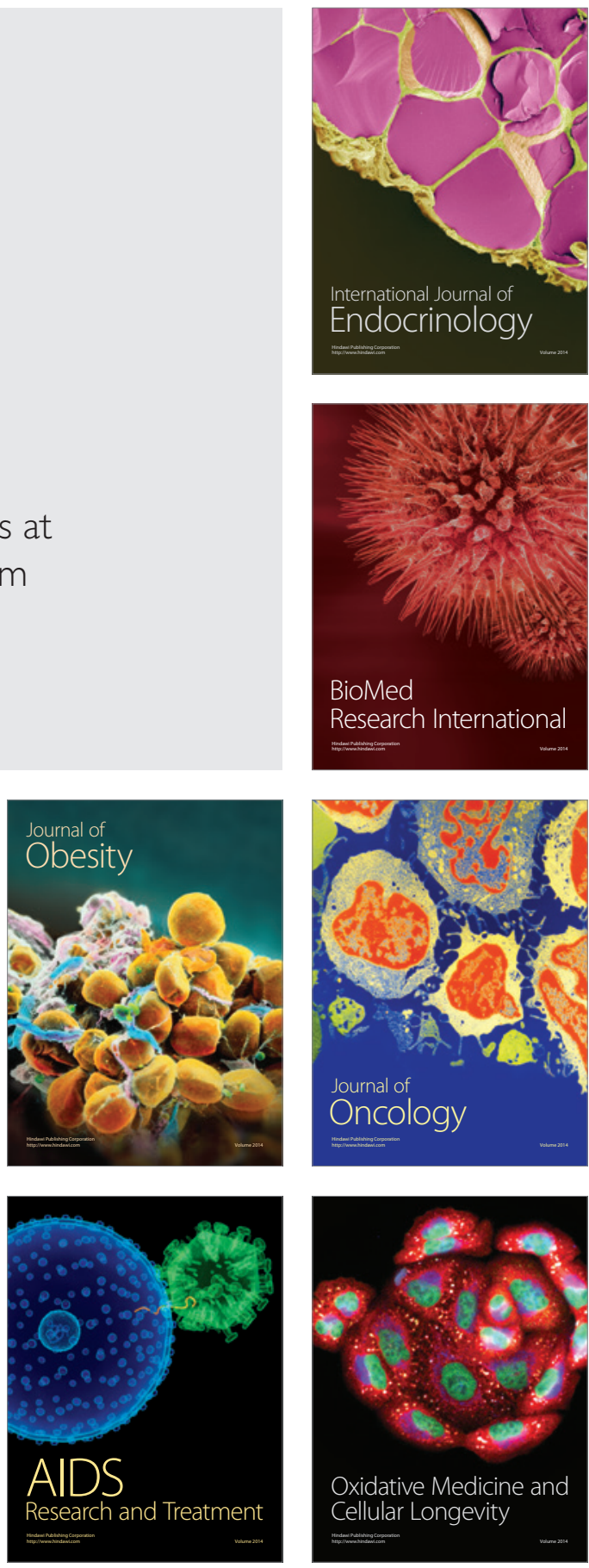